\title{
Ueber Molobrus-Eier,
}

\section{zu Cantagallo in Brasilien von Herrn C. Euler gesammelt. Von}

Ludwig Holtz.

Mit Bezug auf den Sitzungsbericht vom 2. Januar 1871 (siehe Jahrg. 1871, Seite 76) sind mir 4 Exemplare von Molobrus-Eiern, welche Herr C. Euler in diesem Journal, 1867, Seite 415 u. ff. beschrieben hat und zwar 2 der Varietät a und $2 \operatorname{der}$ Varietät $b$ angehörend, sowie zwei von genanntem Herrn gefertigte $\mathrm{Ab}$ bildungen, je 1 Exemplar der beiden Varietäten repräsentirend, zur Vergleichung und Berichterstattung zugesandt worden. Ausserdem einige hierauf bezügliche briefliche Notizen des Herrn Euler.

Bevor ich mich an die gewünschte Beurtheilung mache, verweise ich auf die früheren Aufsätze in diesem Journale, Jahrg. 1867, S. 415 u. ff.; 1869 , S. 125 u. ff., und 1870 , S. $15-18$.

$\mathrm{Zu}$ vergleichen, resp. $\mathrm{zu}$ beschreiben sind nun folgende $\mathbf{M o}$ lobrus-Eier:

I. Gesammelt in der Umgegend von Buenos-Ayres von Chrysanthus Sternberg, beschrieben von Ludwig Holtz - Journ. f. Orn., Jahrg. 1870, S. 15-18.

II. Gesammelt zu Cantagallo in Brasilien von Carl Euler, und zwar:

\section{1) Varietät a.}

a) Beschrieben von C. Euler - Journ. f. Orn., Jahrg. 1867, S. 415 u. 416.

Gestalt: Stark oval, wenig länglich, Vorderende mit sehr sanfter Spitze.

Schale: Mehr rauh, ohne Glanz.

Färbung: Colorit: roth; Grundfarbe: weiss mit lebhaft röthlichem Anfluge; Zeichnung: blassviolette und hellbraunrothe Flecken und Punkte, erstere verwaschen, letztere scharfbegrenzt. Beide Flecken klein, meist zackig, zahlreich, doch in sehr weiten Abständen über das ganze Ei vertheilt, am stumpfen Ende etwas dichter.

Maass: Grosse Achse 0,024 Mm., kleine Achse 0,019 Mm.; Schneidepunkt bei - 0,012 Mm.

b) Beschrieben von mir nach den beiden vorliegenden Eiern. Gestalt: No. 1 - eiförmig-kurz; No. 2 - eigestaltiglänglich. 
Schale: No. 1 - Stärke: fest; Oberfläche porzellanartig, glänzend-glatt; Korn zerstreut punktirt. No. 2 - ebenso.

Färbung: No. 1 - Grundfarbe: weiss mit röthlichem Anfluge; Zeichnung: bestimmt begrenzte, meist ziemlich grosse, vielgestaltige, lebhaft braunröthliche Flecken und einige schwarze Pünktchen mit spärlich dazwischen auftretenden blaugrauen und verwaschenen Flecken. Vertheilung: die Flecken über die ganze Schale zerstreut, die Kuppe freilassend für einige verwaschene Flecken, treten sie an der Basis, zu einem Kranze geformt, am stärksten auf, bis fast zur Querachse sich in derselben Anzahl fortziehend und sich dann weiter strahlenförmig, in immer geringerer Anzahl, bis zur Spitze fortziehend.

No. 2-Grundfarbe: weiss mit gelblichem Anfluge; Zei chnung: bestimmt begrenzte, meist mit einem schwarzen Korn versehene, punktförmige, braunröthliche Flecken, zwischen welchen sich hin und wieder verwaschene violette befinden. Vertheilung: auf der Basis am zahlreichsten auftretend und sich strahlenförmig in immer geringerer Anzahl bis zur Spitze fortziehend.

Maasse: Länge: No. $1-23 \mathrm{Mm}$.; No. $2-23 \mathrm{Mm}$. Breite: No. 1 - $19 \mathrm{Mm}$.; No. $2-17 \mathrm{Mm}$. Durchschnittsmaasse beider Eier:

Länge: Durchschn. = Min. $=$ Max. $=23 \mathrm{Mm}$.

Breite: Durchschn. 18 Mm., Min. 17 Mm., Max. 19 Mm.

Gewicht: Entleert: No. $1-5^{4} / 8$ Gr.; No. $2-5^{7 / 8}$ Gr. Durchschnitt beider : $5^{11 / 16} \mathrm{Gr}$.

2) Varietät b.

a) Beschrieben von C. Euler - Journ. f. Orn., Jahrg. 1867, S. 415 u. 416.

Gestalt: Dickrund, kugelig, beide Enden gleich stumpf.

Schale: Glatt und glänzend.

Färbung: Colorit: grün; Grundfarbe: weisslich-grüu; Zeichnung: gelbbraune Spritzflecken und Punkte, darunter spärlich verwaschene, aschblaue Stellen, Flecken kleiner als bei Varietät a, doch viel dichter und enger, das ganze Ei durchaus gleichmässig bedeckend.

M a a s s e: Grosse Achse 0,0211/2 Mm., kleine Achse 0,018 Mm. ; Schneidepunkt bei $-0,011 \mathrm{Mm}$.

b) Beschrieben von mir nach den beiden vorliegenden Eiern. Gestalt: No. 1 - eiförmig-kurz; No.2 - eiförmig-kurz. 
Schale: No. 1 - Stärke fest; Oberfläche porzellanartig, glänzend-glatt; Korn zerstreut punktirt. No. 2 - ebenso.

Färbung: No. 1 - Grundfarbe: weiss mit grünlichem Anfluge; Zeichnung: unbestimmt begrenzte, feine graubraune und kleine punktförmige, schwarze, mit blaugrauen verwaschene, wechselnde Flecken. Vertheilung: über die ganze Oberfläche des Eies, mit Ausnahme der Spitze, wo eine etwas geringere Anhäufung bemerkbar, gleichmässig und sehr zahlreich angehäuft.

No. 2 - Grundfarbe: weiss mit gelbgrünlichem Anfluge; Zeichnung: bestimmt begrenzte, meist grössere vielgestaltige graubraune, mit in geringerer Anzahl vorhandenen blaugrauen abwechselnden Flecken. Vertheilung: überhaupt nicht sehr zahlreich, an der Basis aber am zahlreichsten auftretend und sich bis zur meist freien Spitze ziemlich gleichmässig in geringerer Anzahl fortziehend.

Maasse: Länge: No. 1 - $21 \mathrm{Mm}$; No. 2 - $19 \mathrm{Mm}$.

Breite: No. $1-17 \mathrm{Mm}$; No. $2-16 \mathrm{Mm}$.

Durchschnittsmaasse beider Eier:

Länge: Durchschn. 20 Mm., Min. 19 Mm., Max. 21 Mm.

Breite: Durchschn. $16 \%$ Mm., Min. 16 Mm., Max. $17 \mathrm{Mm}$.

Durchschnittsmaasse der 4 Eier der Variationen a und b:

Länge: Durchschn. $22 \mathrm{Mm}$, Min. $19 \mathrm{Mm}$, Max. $23 \mathrm{Mm}$. Breite: Durchschn. 171/4 Mm., Min. 16 Mm., Max. 19 Mm.

Gewicht: Entleert: No. $1-4 \frac{4}{8}$ Gr.; No. $2-4 \frac{1}{8}$ Gr. Durehschnitt beider $4 \frac{5}{16} \mathrm{Gr}$.

Durchschnittsgewichte der 4 Eier der Varietäten $a$ und $b$ :

Durchschn. 5 Gr., Min. $4^{1 / 1}$ Gr., Max. $5^{7 / 8}$ Gr.

Vergleichen wir nun:

I. Varietät a nach beiden Beschreibungen;

II. Varietät $b$ nach beiden Beschreibungen;

III. Varietät a mit Varietät $b$ mit Zugrundelegung der Beschreibungen;

IV. beide C. Euler'schen Varietäten mit den von Chrysanthus Sternberg gesammelten und von mir beschriebenen.

\section{Varietät a.}

1) Gestalt: Keine wesentlichen Unterschiede.

2) Schale: Beide Beschreibungen differiren sehr; indess be- 
richtigt. C. Euler seine frühere Beschreibung in dem angegebenen Briefe d. d. Basel, den 27. October 1870 dahin:

„Was nun die Schale anbetrifft, so kann ich mir heute nicht erklären, warum ich früher Varietät a ohne Glanz bezeichnete, das damals vorliegende Exemplar mag daran schuld sein, denn in ihrem jetzigen Zustande sind die beiden Varietäten in Bezug auf Glanz ziemlich gleichzustellen."

Also keine Unterschiede.

3) Färbung:

Grundfarbe: Mit „lebhaft röthlichem Anfluge“ und „mit röthlichem Anfluge".

C. Euler schreibt in dem angegebenen Briefe:

„H. Holtz glaubt den „lebhaft röthlichen Anflug“ bei Varietät a in der "Frische" der bezüglichen Eier motiviren zu müssen, und scheint überzeugt zu sein, derselbe werde durch's Aufbewahren verschwinden. Besonders das eine Exemplar obiger 4 Eier wird Ihnen beweisen, dass die rothe Nüance nicht flüchtig, sondern sehr solide ist, denn diese Eier befinden sich schon seit 5 Jahren in meiner Sammlung. Dasselbe kann von dem grünlichen Ton der Variatät b gelten. Die Abbildungen zeigen Ihnen auf's genaueste die beiden Nüancen der frischen Eier. Vielleicht hat der von mir gebrauchte „Anflug“ Herrn Holtz zu seiner Annahme verleitet, ich hätte füglich sagen können: "Grundfarbe hellroth, bald kräftiger, bald schwächer"."

Ich muss hierzu erwähnen, dass ich bei der Vergleichung der Chr. Sternberg'schen Eier mit den C. Euler'schen Varietäten Journ. f. Orn., Jahrg. 1870, S. 16 - durch das "sein dürfte“ nur eine Vermuthung habe aussprechen wollen - verändern doch Mergus serrator und Anas fusca leicht ihre ursprüngliche Grundfarbe - und bin nun vollkommen überzeugt, dass die rothe Nüance nicht flüchtig ist. Indess kann ich doch bei dem einen vorliegenden Exemplare der Grundfarbe einen lebhaft röthlichen Anflug nicht anerkennen, sondern meine schon genug gethan zu haben, wenn ich röthlich sage. Doch soll das gerade nicht heissen: „es ist überhaupt", sondern nur: „es ist nach meiner Meinung“, denn es ist und bleibt immerhin schwierig, die feinen Nüancirungen der Farben durch den Blick und die entsprechenden Worte zu constatiren.

Es macht hier auch keinen wesentlichen Unterschied. Bei No. 2 habe ich die Grundfarbe: weiss mit gelblichem An- 
fluge angegeben, während C. Euler überhaupt bei seiner Varietät a nur von „weiss mit röthlichem Anfluge" spricht.

Ich erinnere hier an die verschiedenen Färbungen der $A n$ thus arboreus, der Lariden und Sterniden, wodurch keine specifis chen Unterschiede constatirt werden.

Zeichnung: Bezüglich der Grösse der Flecken sagt C. Euler „beide Flecken klein“, während meine Beschreibung von No. 1 von "meist ziemlich grossen" und von No. 2 von "punktförmigen" handelt, wodurch indess keine wesentlichen Unterschiede festgestellt werden.

Vertheilung: Keine wesentlichen Unterschiede.

4) Maass: Keine wesentlichen Unterschiede.

II. $V$ ariet ät $b$.

1) Gestalt: Keine Unterschiede.

2) Schale: Keine Unterschiede.

3) Färbung:

Grundfarbe: Bei No. 1 und 2 keine wesentlichen Unterschiede.

Zeichnung: Während C. Euler hinsichtlich der Grösse der Flecken sagt: „Flecken kleiner als bei Varietät a", lautet meine Beschreibung bei No. 1 „punktförmige" und bei No. 2 "meist grössere"; also keine wesentlichen Unterschiede.

Vertheilung: Mit No. 1 übereinstimmend, doch nicht ganz mit No. 2 ; doch keine wesentlichen Unterschiede.

4) Maass: Bei No. 1 kein wesentlicher Unterschied; ebenso wird auch durch die etwas geringere Grösse von No. $2 \mathrm{ke}$ in wesentlicher Unterschied festgestellt.

III. Varietät a mit Varietät b.

1) Gestalt: Bezüglich der Gestalt und Grösse schreibt C. Euler in dem oben angegebenen Briefe:

"Bei Form und Gröss e findet H. Holtz Uebereinstimmung, d. h. aber mit den Eiern des H. Sternberg. Denn das muss ich wiederholt betonen, dass meine beiden Varietäten besonders in Grösse und auch meistens in Form constante Verschiedenheiten unter einander aufweisen. Varietät a ist beständig grösser als Varietät $\mathrm{b}$ und meistens auch schlanker, während b oft kurz kugelig vorkommt."

$\mathrm{Da}$ in beiden Varietäten nach meiner Beschreibung sowohl die eiförmige als eigestaltige Form auftritt, so kann ich, bezüglich der Gestalt, keine wesentlichen Unterschiede constatiren. 
2) Schale: Keine Unterschiede.

3) Färbung:

Grundfarbe: Wenn auch die Grundfarbe der unter Varietät a aufgeführten Eier mit „weiss mit lebhaft röthlichem Anfluge weiss mit röthlichem Anfluge - weiss mit gelblichem Anfluge" aufgeführt worden, während die Grundfarben der Varietät b mit „Weisslichgrün - weiss mit grünlichem Anfluge - weiss mit gelbgrünlichem Anfluge" bezeichnet sind; so werden dadurch doch keine wesentlichen Unterschiede festgestellt.

Ich erinnere hier wieder an die verschiedenen Grundfarben von Anthus arboreus, der Gattungen Larus, Sterna u. a.

Zeichnung: C. Euler schreibt in dem angegebenen Briefe:

„Wie bei der Grundfarbe, so scheinen mir auch die Flecken wirklich in ihrer Farbe verschieden zu sein, wenigstens was die braunen anbetrifft. Bei Varietät a sind sie entschieden: rothbraun, bei Varietät b g elbbraun."

Stellen wir zur besseren Uebersicht die Farbenzeichnungen der Flecken nach den Beschreibungen nebeneinander, so haben wir bei

$\begin{array}{ll}\text { Varietät a: } & \text { Varietät b: } \\ \text { rothbraun } & \text { gelbbraun. } \\ \text { hellbraunroth } & \text { - gelbbraun. } \\ \text { braunröthlich } & - \text { graubraun. }\end{array}$

Es sind lauter Nüancirungen von braun, keine, hier in Betracht kommenden wesentlichen Unterschiede vorhanden.

Hinsichtlich der Grösse der Flecken, so ist auch eine Gleichheit derselben in je einer der Varietäten nicht durchstehend; sondern es zeigen die Schalen von zwei Eiern, welche beiden Varietäten angehören: grosse, während die anderen zwei, gleichfalls beiden Varietäten angehörende kleine Flecken zur Schau stellen.

Also keine wesentlichen Unterschiede vorhanden.

Bezüglich der Zeichnung erinnere ich hier an die Eier von Lanius collurio, über welche sich schon mancher Ornithologe den Kopf zerbrochen und worüber die Acten noch nicht geschlossen sind.

4) Masse. Es betragen die Durchschnittsmaasse:

$$
\text { Länge }
$$

bei Varietät a: Varietät $b$ :

$23 \mathrm{Mm}$.

$20 \mathrm{Mm}$.

Varietät a:

Breite

Es möchten hier Unterschiede in Betracht zu ziehen sein. 
IV. Die C. Euler'schen Varietäten a und b mit den von Chrys. Sternberg gesammelten Eiern.

1) Gestalt: Keine Unterschiede.

2) Schale: Keine Unterschiede. - Vergl. Aufsatz I, 2.

3) Färbung: Ausser den völlig weissen Eiern keine Unterschiede.

4) Maasse: Zur besseren Uebersicht nachstehende Tabelle.

\begin{tabular}{|c|c|c|c|c|c|c|}
\hline \multicolumn{7}{|c|}{ Uurchschnittsmaasse. } \\
\hline \multirow[b]{2}{*}{$\begin{array}{c}\text { Eier, } \\
\text { gesammelt } \\
\text { von: }\end{array}$} & \multicolumn{3}{|c|}{ Länge. } & \multicolumn{3}{|c|}{ Breite. } \\
\hline & $\begin{array}{c}\text { Durch- } \\
\text { schnitt. } \\
\text { Mm. }\end{array}$ & $\begin{array}{c}\text { Minimal. } \\
\mathrm{Mm} .\end{array}$ & $\begin{array}{c}\text { Maximal } \\
\mathrm{Mm} .\end{array}$ & $\begin{array}{c}\text { Durch- } \\
\text { schnitt. } \\
\text { Mm. }\end{array}$ & $\begin{array}{c}\text { Minimal. } \\
\mathrm{Mm} .\end{array}$ & $\mathrm{Mm}$ \\
\hline $\begin{array}{l}\text { Chrys } \\
\text { ber }\end{array}$ & 61. & 20. & 24. & 1 & 16. & \\
\hline $\begin{array}{l}\text { Euler, } \\
\text { Var. a v }\end{array}$ & & 19. & 20 & $171 / 4$. & 16. & 19 \\
\hline
\end{tabular}

Also keine wesentlichen Unterschiede vorhanden.

Es haben sich überhaupt nach den vorstehenden Vergleichungen, mit Ausnahme von III, 4, keine Unterschiede herausgestellt. Das Gewicht habe ich hier überhaupt nicht in Betracht gezogen, da gar leicht mangelhafte Entleerung und zu grosse Oeffnungen, wie hier hin und wieder sich auch herausstellen, grosse Differenzen, also falsche Resultate ergeben.

Bevor ich nun in den Untersuchungen weiter fortfahre, führe ich noch aus dem C. Euler'schen Briefe an:

"Was ich früher schon constatirte und heute wiederhole, ist: dass beide Varietäten sehr beständig in oben angeführten Untërschieden auftreten, dass mir bis jetzt keine entschiedenen Uebergänge vorgekommen sind und dass Varietät $\mathbf{b}$ das authentische Ei von Mol. sericens ist, weil ich diesen Vogel selbst aus dem betreffenden Ei gross gezogen. Die Mol.-Eier fand ich (beide Varietäten) ziemlich gleich häufig bis jetzt nur in offenen Napfnestern und zwar überwiegend bei Zonotrichia matutina, bei Höblenbrütern, wie Troglodytes platensis, nie. - Anders gestaltete oder gefärbte Molobrus-Eier, als die beschriebenen Varietäten a und b, habe ich in Cantagallo nicht kennen gelernt. Die weissen, ungeHleckten Eier des Herrn Sternberg habe ich dorten nie gefunden." Wenn ich mir nun die 4 übersandten, 2 Varietäten repräsen- 
tirenden Molobrus-Eier anschaue, von welchen C. Euler - Journ. f. Orn., Jahrg. 1867, S. 416, unten - sagt:

„Bis dahin nehme ich an, dass die beiden oben beschriebenen Varietäten zwei verschiedenen Species angehören, denn die Unterschiede in Farbe und Gestalt scheinen mir zu gross $\mathrm{zu}$ sein, um beide dem gleichen Vogel zuschreiben zu dürfen," und ich über kein anderes betreffendes Material zu verfügen hätte, so möchte ich auch wohl geneigt gewesen sein, zu glauben, dass ich 2 verschiedene Arten vor mir hätte, und kann ich mich sehr wohl in C. Euler's Stelle versetzen.

Vergleiche ich aber dảnn die 4 Eier mit meinen 50 Exemplaren, reihe die ersten den anderen wechselsweise an, um Formübergänge auszugleichen, und sehe, welche Mannigfaltigkeit in den Formen und Färbungen, aber immer durch Uebergänge vermittelt, obwaltet, dagegen welche Gleichheit in der Structur der Schale sich herausstellt, so muss ich die feste Vermuthung aufstellen, dass wires hier nur mit einer Art zu thun haben.

Wie ich schon bei den verschiedenen Vergleichungen hin und wieder erwähnt, so herrscht oft bei den Eiern einer Art die grösste Mannigfaltigkeit der Formen - Larus, Sterna u. a. - , der Grundfarben - dieselben und Anthus arboreus -, der Zeichnungen dieselben und Lanius collurio u. a., sowie auch der Grösse.

Bezüglich letzterer möchte zu III. 4 noch eine Aufklärung, wenigstens Erörterung nöthig sein.

Also nehmen wir an: es kann ja sehr leicht 2 Arten geben, die sich nur ganz unmerklich durch die Farbe des Gefieders unterscheiden, gleiche Grösse haben und dann leicht dem Auge entgehen können; indess hier, wo die constante Grösse einer kleineren Eierform in Betracht kommen soll, da muss es auch eine constante kleinere Art geben; und sollte die bei dem häufigen Vorkommen des Molobrus sericeus von den Ornithologen bis dahin wohl übersehen sein; besonders da dieselbe constante kleinere Eierform nach C. Euler - Journ. f. Orn., Jahrg. 1867, S. 416 erster Absatz ungefähr in gleicher Anzahl mit der grösseren gefnnden wird? Ich glaube nicht. klärung.

Es ist mir aber Eines sehr merkwürdig und bedarf der Auf-

C. Euler schreibt: - Journ. f. Orn., Jahrg. 1867, S. 416 - 
„Varietät a muss ich als das authentische Ei von Icterus violaceus bezeichnen u. s. w.,"

und ferner in der soeben angegebenen Stelle des Briefes:

„.... dass mir bis jetzt keine entschiedenen Uebergänge vorgekommen sind, und dass Varietät $\mathbf{b}$ das authentische Ei von Molobrus sericeus ist u. s. w."

Da nun die hier angeführten Var. a und b sowohl mit den, in natura übersandten Eiern, als auch mit den, auf der beigefügten Tafel, mit Var. und b bezeichneten, gemalten Eiern übereinstimmend sind, nach Dr. Cabanis - Journ. f. Orn., J. 1870, S. 17* sericeus = violaceus, so würde daraus zu schliessen sein, dass beide Var. nur eine Art repräsentirten, obgleich C. Euler das Gegentheil klar ausspricht.

Es muss da meines Erachtens wohl ein Versehen oder Irrthum vorliegen.

Um Untersuchungen anzuregen, will ich noch eine Bemerkung, bezüglich der Färbung der mir vorliegenden Molobrus-Eier hinzufügen. Unter den Sternberg'schen Eiern befinden sich fast e be n so viel weisse ohne Zeichnung als mit Zeichnung, dagegen befinden sich unter einigen 50 nur 4 Eier weiss mit grünlichem Anfluge, während nach C. Euler die weissen nie in Cantagallo, dagegen eben so viele mit weiss grüner Grundfarbe als mit der andern Färbung gefunden werden.

Ich erinnere hier wieder an Lanius collurio, es möchten gleiche Gründe für die verschiedenen Färbungen vorhanden sein.

Schliesslich möchte ich noch bemerken, dass ich durchaus nicht etwa einen Streit heraufbeschwören will, dass ich meine Behauptungen nicht unterschreibe mit: „von Rechtswegen, es ist so!“ Ich nehme sehr gern Belehrungen an, da nur durch gegenseitige Aussprache die Wissenschaft gefördert werden kann.

Barth, den 18. März 1871.

\section{Die sibirischen Laubvögel. \\ Von}

\section{E. F. v. Homeyer.}

Durch die grosse Güte des Herrn L. Taczanowski ist mir eine Anzahl sibirischer Laubvögel zu Händen gekommen, so dass ich aus eigener Anschauung darüber urtheilen kann. Leider ist es mir unmöglich gewesen, die zahlreichen indischen Arten damit zu vergleichen, indem dieselben kaum in einer der Sammlungen, 

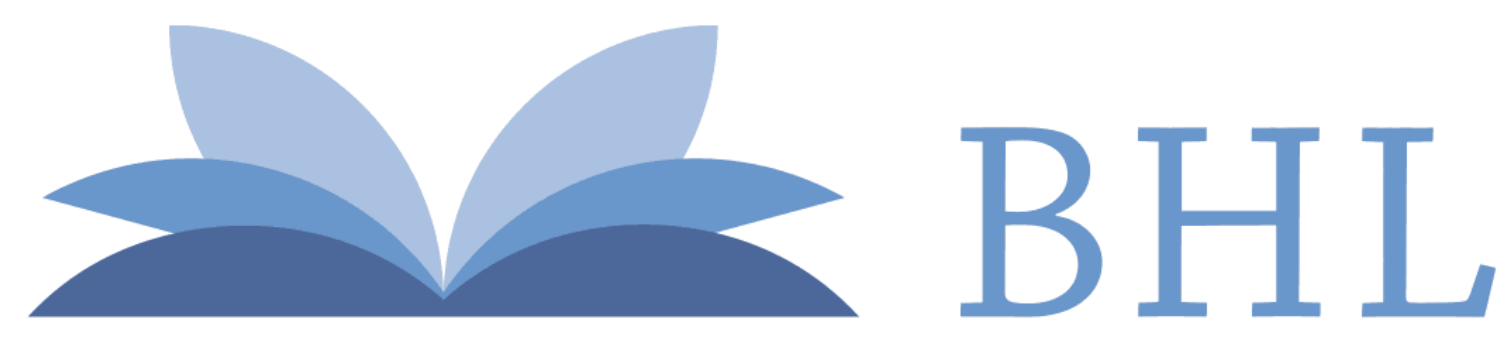

\section{Biodiversity Heritage Library}

1872. "Ueber Molobrus-Eier, zu Cantagallo in Brasilien von Herrn C. Euler gesammelt." Journal

$f u$

r Ornithologie 20, 193-201. https://doi.org/10.1007/bf02252711.

View This Item Online: https://www.biodiversitylibrary.org/item/102848

DOI: https://doi.org/10.1007/bf02252711

Permalink: https://www.biodiversitylibrary.org/partpdf/141889

\section{Holding Institution}

Smithsonian Libraries

\section{Sponsored by}

Biodiversity Heritage Library

\section{Copyright \& Reuse}

Copyright Status: Public domain. The BHL considers that this work is no longer under copyright protection.

This document was created from content at the Biodiversity Heritage Library, the world's largest open access digital library for biodiversity literature and archives. Visit BHL at https://www.biodiversitylibrary.org. 